\title{
Oncogene EGF
}

National Cancer Institute

\section{Source}

National Cancer Institute. Oncogene EGF. NCI Thesaurus. Code C18494.

Human Oncogene EGF is a mutated variant of EGF Gene, which encodes Epidermal Growth Factor, a 53-aa peptide hormone with profound effects on cell differentiation and potent mitogenic effects on a variety of ectodermal and mesodermal cells. The EGF precursor is believed to exist as a membrane-bound molecule that is proteolytically cleaved to generate the hormone. Oncogene EGF disrupts normal cell function. 